Article

\title{
LEED-EB Gold Projects for Office Spaces in Large Buildings Transitioning from Version 3 (v3) to 4 (v4): Similarities and Differences between Finland and Spain
}

\author{
Svetlana Pushkar $\mathbb{D}$ \\ Department of Civil Engineering, Ariel University, Ariel 40700, Israel; svetlanap@ariel.ac.il; Tel.: +972-3-9066-410
}

Received: 23 October 2020; Accepted: 3 December 2020; Published: 6 December 2020

\begin{abstract}
This study aims to assess the similarities and differences between Finland and Spain in terms of Leadership in Energy and Environmental Design for Existing Buildings (LEED-EB) Gold large office building-type projects transitioning from version $3(\mathrm{v} 3)$ to version $4(\mathrm{v} 4)$. The percentages of the average scores are used here to assess the achievements of the LEED-EB data. The natural logarithm of the odds ratio $(\ln \theta)$ and Fisher's exact $2 \times 2$ tests with a mid $p$-value are used to evaluate dichotomous data, while the exact Wilcoxon-Mann-Whitney and Cliff's $\delta$ effect size tests are used to evaluate ordinary data. The results for LEED-EB Gold large offices demonstrate similar certification strategies in Finland and Spain. These results may be useful to LEED-EB practitioners in Finland and Spain for facilitating the selection of appropriate certification strategies in line with identified high-performance credits for large offices.
\end{abstract}

Keywords: LEED-EB v3; LEED-EB v4; Europe

\section{Introduction}

In 1998, the United States Green Building Council (USGBC) initiated the Leadership in Energy and Environmental Design (LEED) as the U.S. rating system for building sustainability certification, which has been continually improved from version to version [1]. LEED can currently be applied to various building types, such as LEED for New Construction and Major Renovations (LEED-NC), Commercial Interiors (LEED-CI), Core and Shell Development (LEED-C-and-S), and Existing Buildings Operations and Maintenance (LEED-EB), among others. These systems evaluate the following categories: location and transportation (LT), sustainable sites (SS), water efficiency (WE), energy and atmosphere (EA), materials and resources (MR), indoor environmental quality (EQ), innovation and design (ID), and regional priority (RP). Each of these categories contains anywhere from one to several credits. These credits prescribe the relevant requirements and award different numbers of points, according to the importance of a given issue in building-related sustainability. As a result, buildings can be certified as follows: Certified (40-49 points), Silver (50-59 points), Gold (60-79 points), or Platinum (80 points and above).

LEED is also well-known as an international system, as it is in demand in many countries around the world [2]. In Europe, LEED is popular in such countries as Italy, Turkey, Spain, Finland, and Sweden [3]. At the same time, LEED has often been criticized for its non-adaptability to foreign countries, as they have different climates, demographics, technologies, and cultural features than the U.S., for which LEED was initially created [4]. As a result, LEED introduced regional priority points and alternative compliance paths for East Asia, Europe, and South America [5].

With the flexibility of the LEED system, each project can choose an appropriate certification strategy based on its social, environmental, and economic goals. This process is a challenge, due to the 
relationship between LEED achievements and the various factors that influence certification, such as local green policy, the project-related certification target, budget, schedule, location, climate, building type, and area [6]. It is believed that research support for these factors could help LEED managers to achieve the necessary level of certification in the limited framework of a project's budget and schedule constraints [7]. Thus, the publication of foreign country-specific LEED certification strategies is essential, as these publications reflect appropriate sustainable strategies in local contexts. In this respect, recent research has shown that foreign countries can find appropriate LEED certification strategies.

For example, Wu et al. have analyzed certified projects relevant to the U.S., Turkey, Brazil, and China [5]. For each of these countries, the authors evaluated their LEED-NC version 3 (v3) Certified, Silver, Gold, and Platinum projects together in one pooled set. The authors found different certification strategies in these countries. For example, the U.S. showed better results than China in the EA category, whereas China showed better results than the U.S. in the SS and WE categories [5]. Thus, the results presented somewhat averaged strategies for the analyzed countries and seemed to not be very indicative for each of the certification levels [8]. This is due to the different certification strategies that are usually applied to high levels (Gold and Platinum) and low levels (Certified and Silver) [9].

Toğan and Thomollari studied LEED-NC v3 projects certified in Turkey [10]. They concluded that the credit achievement rates in the various categories were the same as that of $\mathrm{Wu}$ et al. [5]. However, the authors evaluated the different spatial types of LEED-NC v3 projects, such as commercial offices, basic training, data centers, and industrial and residential buildings, among others, as one combined set. Pushkar studied four countries, namely Finland versus Sweden (northern Europe) and Turkey versus Spain (southern Europe) [11]. In each of these countries, only LEED-NC v3 Gold projects were evaluated. The author revealed different certification strategies for these countries [11]. For example, in terms of outdoor water saving, Sweden showed better results than Finland, while Spain showed better results than Turkey. However, the author analyzed different types of LEED-NC v3 Gold projects, such as in offices, multi-family residential buildings, industrial manufacturing buildings, public assembly buildings, healthcare buildings, higher education buildings, and data centers, among other buildings, considering every project in one pooled set [11]. Combining such LEED projects with different properties into one group, as in [10] and [11], may lead to incorrect conclusions [8].

For example, healthcare buildings may be more interested in the performance of the EQ category than data centers. An additional example is the installation of different heating, ventilation, and air conditioning (HVAC) systems in small and large offices; small offices usually use decentralized systems (DXs), whereas large offices usually install central HVAC systems [12]. Thus, different HVAC systems may lead to different performance results for the EA category between small and large offices.

Pham et al. studied LEED-NC v3 certification strategies in Vietnam [13]. The authors analyzed three certification levels and revealed that several credits showed different values between the LEED Silver and LEED Gold projects, as well as between the LEED Gold and LEED Platinum projects. It was concluded that the revealed LEED certification strategies reflected specific characteristics of Vietnam, including the climatic conditions (i.e., a humid tropical climate). However, building types were not specified in their study [13]. Pushkar focused on three Mediterranean countries: Turkey, Spain, and Italy [14]. The author studied LEED-CI v3 and LEED-C-and-S v3 Gold projects. These countries are located in areas with similar regional environmental concerns. However, the author found similar performance results between the countries, in terms of the SS, MR, and EQ credits, whereas the WE and EA credits showed different values between these three countries [14]. In this study, building types were not specified. Thus, the certification strategies revealed by Plam et al. [13] and Pushkar [14] may also suffer from inaccuracies.

All of the aforementioned LEED foreign-related studies [5,9-11,13,14] can be suspected of incorrect data collection processes and, thereby, should be treated with some caution. Moreover, these studies have mostly analyzed newly constructed buildings. However, in Europe, the most urgent issue is the retrofitting of existing buildings, as these represent the main building stock [15]. Retrofitting is a more sustainable process than the construction of new buildings [16]. This is because performing retrofitting 
by improving insulation and HVAC effectiveness results in less materials being required and less waste generation [17]. However, retrofitting also presents many risks and uncertainties related to the selected retrofitting techniques and approaches, restricted financial and schedule frames, and the significant energy saving payback period [18].

The literature describes several cases of successful implementations of LEED certification in building retrofits. For example, Dall'O et al. analyzed the option of improving school sustainability in northern Italy through the application of LEED-NC v3 requirements [19]. Sun et al. studied the Chow Yei Ching (CYC) University building in Hong Kong which, in 2015, received LEED-EB v3 Gold certification after the application of energy efficient retrofit measures [20]. Mazzola et al. used LEED-EB version $4(\mathrm{v} 4)$ certification to suggest green retrofit measures for a museum (Ca' Rezzonico) in Italy [21]. Thus, green certification is useful, as it prescribes directions and tools toward the sustainable retrofitting of existing buildings [22].

In addition, all the aforementioned LEED foreign-related studies analyzed only the projects certified under the previous version (v3) of LEED certification $[5,9-11,13,14]$. However, the current version ( $\mathrm{v} 4$ ) of LEED [23] certification differs in several respects from its predecessor [24]. This is the case for all LEED systems, including LEED-EB. Thus, in LEED-EB, the SS category of version 3 was reformulated into the LT and SS categories in version 4, and some credits were reformulated and re-evaluated (in terms of their maximum possible points) in version 4, compared with their evaluation in version 3 (e.g., the EQc5 Daylight and Quality Views credit has four maximum awarded points, compared with the EQc2.4 Daylight and Views credit in version 3, which has only one maximum awarded point). Thus, there is a different distribution of maximum possible points in LEED v3 and $v 4$ categories. In particular, LEED v3 includes SS (26 points), WE (14 points), EA ( 35 points), MR (10 points), and EQ (15 points) categories [24], whereas LEED v4 includes LT (15 points), SS (10 points), WE (12 points), EA (38 points), MR (8 points), and EQ (17 points) categories [23]. Thus, for more precise evidence for changing the certification strategies in LEED foreign-based countries, versions 3 and 4 need to be evaluated in parallel. However, no such empirical research — that is, research which analyzes the same building type of LEED-EB v3 and v4 projects in Europe-has yet been conducted.

Thus, the present study aims to fill this gap by studying European LEED-EB v3 and v4 certifications for large office buildings in Finland (northern Europe) and Spain (southern Europe).

The main research questions are the following. Are there similarities or differences in the certification strategies of the same building type (i.e., large offices) in countries with similar, European-based community values and different natural energy and water resources, as well as different climate conditions? Have these similarities or differences changed when improving from LEED-EB version 3 to version 4? It is worth noting that only LEED-EB v3 and v4 projects at the Gold level have a sufficient sample size to statistically assess differences between Finland and Spain, so only Gold projects are considered here.

\section{Materials and Methods}

\subsection{Methodology Flowchart of the Present Study}

Figure 1 shows the three sequential steps for data collection and two types of data analysis. Data collection and analysis procedures are presented in Sections 2.2 and 2.3. 


\section{Data colection}

Collect LEED-EB v4 Gold large office-type $\left(>6435 \mathrm{~m}^{2}\right)$ projects in Finland and Spain and record the following properties: Number of projects, city, and last certification time.

Collect LEED-EB v3 Gold large office-type $\left(>6435 \mathrm{~m}^{2}\right)$ projects in Finland and Spain

with the same properties as identified in LEED-EB v4.

This design structure is used to reduce the influence of uncontrollable factors when two independent groups are to be compared.

\section{Data analysis}

When the LEED had dichotomous data, natural log odds ratio and Fisher's exact test $2 \times 2$ with mid-p-value were used to the estimate the effect size and P-value, respectively.

When LEED had ordinary data, the Cliff's $\sigma$ and exact Wilcoxon-Mann - Whitney tests were used to estimate the effect size and P-value, respectively.

Figure 1. The Leadership in Energy and Environmental Design (LEED) version 3 (v3) and version 4 (v4) Gold large office-type projects, using a top-down research framework to evaluate similarities and differences between Finland and Spain.

\subsection{Data Collection}

The achieved credits of LEED-EB v3 and v4 Gold projects were collected from the USGBC office site [25], while space types and project sizes were collected from the Green Building Information Gateway (GBIG) global platform [26]. The USGBC and GBIG databases discovered 14 LEED v4 Gold large office-type $\left(>6435 \mathrm{~m}^{2}\right)$ projects in Finland and 16 in Spain. Furthermore, the following properties were recorded: the number of projects, the city, and the last certification time $[25,26]$. Based on the USGBC and GBIG databases, we collected the same number of LEED-EB v3 large office-type $\left(>6435 \mathrm{~m}^{2}\right)$ projects with the same properties, as identified in LEED-EB v4 $[25,26]$. This design structure was used to reduce the influence of uncontrollable factors when two independent groups were to be compared [27,28]. Table 1 demonstrates that, for both versions, the differences between building sizes certified in Finland and Spain seemed to be negative. Table 2 shows that versions 3 and 4 of the LEED-EB Gold projects showed a similar distribution among cities in Finland and Spain.

Table 1. Building size $\left(\mathrm{m}^{2}\right)$ of the Leadership in Energy and Environmental Design for Existing Buildings (LEED-EB) Gold large office-type projects.

\begin{tabular}{|c|c|c|c|}
\hline \multirow{2}{*}{ LEED-EB } & \multicolumn{2}{|c|}{ Median (25th-75th Percentiles) } & \multirow{2}{*}{$p$-Value } \\
\hline & Finland & Spain & \\
\hline v3 & $24,078(14,562-26,311)$ & $18,532(15,596-23,694)$ & 0.5725 \\
\hline $\mathrm{v} 4$ & $18,084(9696-30,282)$ & $14,161(9555-15,668)$ & 0.1794 \\
\hline
\end{tabular}

The $p$-values were evaluated according to the following three-valued logic: seems to be positive (bold font); seems to be negative (ordinary font size); and judgment is suspended (italic font).

Table 2. Distribution of LEED-EB v3 and v4 Gold large office-type projects among cities in Finland and Spain.

\begin{tabular}{cccc}
\hline \multicolumn{2}{c}{ Finland } & \multicolumn{2}{c}{ Spain } \\
\hline Version 4 & Version 3 & Version 4 & Version 3 \\
\hline Helsinki (8) & Helsinki (4) & Madrid (8) & Madrid (9) \\
Espoo (3) & Espoo (4) & Barcelona (7) & Barcelona (4) \\
Oulu (2) & Oulu (2) & Las Rozas (1) & Alcobendas (2) \\
Tampere (1) & Tampere (3) & & Cornellà de Llobregat (1) \\
& Vantaa (1) & Total (16) & Total (16) \\
Total (14) & Total (14) & & \\
\hline
\end{tabular}




\subsection{Data Analysis}

The percentage of average score (PAS) - the ratio of achieved points to maximum points (expressed as a percentage)—was used to assess the performance of LEED data [29]. If the LEED data had a dichotomous structure, then the natural logarithm of the odds ratio $(\ln \theta)$ [30] and Fisher's exact $2 \times 2$ tests were used [31]. If the LEED data had an ordinal structure, then Cliff's $\delta$ effect size [32] and exact Wilcoxon-Mann-Whitney (WMW) [33] tests were used. These non-parametric tests were used as the assumption of normality was not met [34].

\subsubsection{Effect Size Interpretation}

If $\ln \theta$ was zero, then there was no association between the LEED points and Finland or Spain. The left and right limits of $\ln \theta$ were infinite (i.e., the function could change infinitely in either direction away from zero) [35]. Consequently, a value of $\ln \theta$ moving away from zero reflected a stronger relationship between the LEED points and Finland or Spain.

The non-parametric Cliff's $\delta$ test was applied to measure the magnitude of the difference of the two distributions (i.e., the effect size) [30]. Cliff's $\delta$ ranged between -1 and +1 ; positive $(+)$ values indicated that the Finland LEED data was larger than the Spain LEED data, zero indicated an equality or overlap, and negative (-) values indicated that the Spain LEED data was larger than the Finland LEED data [30].

Table 3 shows the absolute effect size thresholds (small, medium, and large) for $|\ln \theta|$ and Cliff's $\delta$.

Table 3. The absolute effect size thresholds.

\begin{tabular}{ccccc}
\hline Effect Size Estimation Procedure & Small & Medium & Large & Reference \\
\hline The absolute natural log odds ratio $|\ln \theta|$ & 0.51 & 1.24 & 1.90 & {$[36]$} \\
The absolute Cliff's $\delta|\delta|$ & 0.147 & 0.33 & 0.474 & [37] \\
\hline
\end{tabular}

The three levels for effect size thresholds had the following definitions. "A medium effect is visible to the naked eye of a careful observer. A small effect is noticeably smaller than medium but not so small as to be trivial. A large effect is the same distance above the medium as small is below it" [38]. It is worth noting that the effect size did not constitute "iron-clad criteria" [39], but was only a general rule of thumb that may be followed in the absence of knowledge of the area [40].

\subsection{2. $p$-Value Interpretation}

A Neo-Fisherian significance assessment (NFSA) was used instead of the paleo-Fisherian and Neyman-Pearson paradigms (i.e., null hypothesis significance tests) [41]. The NFSA paradigm includes the following definitions: (1) the paradigm does not fix $\alpha$ (i.e., the level of significance); (2) the paradigm does not describe $p$-values as significant or nonsignificant; (3) the paradigm does not accept null hypotheses based on high $p$-values, but only suspends judgment; (4) the paradigm presents effect size information in conjunction with $p$-values; and finally, (5) the NFSA paradigm interprets $p$-values based on three-valued logic; namely, either they seem to be positive (i.e., there seems to be a difference between Finland and Spain), seem to be negative (i.e., there does not seem to be a difference between Finland and Spain), or judgment is suspended (regarding the difference between Finland and Spain) [41].

Hurlbert and Lombardi cited Fischer's philosophical proposal that "no scientific worker has a fixed level of significance at which from year to year, and in all circumstances, he rejects [null] hypotheses; he rather gives his mind to each particular case in light of his evidence and ideas." [41] (p. 316). In addition, Beninger et al. noted that the logic of Occam's razor should not be used for universal interpretation of the $p$-value [42]. Therefore, the neo-Fisherian paradigm was used to interpret the signs and magnitudes of the statistical effects [41]. 


\section{Results}

\subsection{Sustainable Sites (SS) and Location and Transportation (LT) Credits and Categories}

Table 4 shows the results for the SS and LT credits and categories. According to these results, in Spain, SSc4 (Alternative Commuting Transportation in version 3) and LTc1 (Alternative Transportation in version 4) had high PAS values (80 and 89, respectively). In contrast, Finland had a low PAS (44) in version 3 of the certification. However, in version 4 of the certification, Finland improved its achieved score a lot $(\mathrm{PAS}=72)$.

Table 4. Statistical analysis of achieved points in the Sustainable Sites (SS) credits for LEED-EB v3 Gold large office-type projects and the Location and Transportation (LT) and SS credits for LEED-EB v4 Gold large office-type projects in Finland and Spain.

\begin{tabular}{|c|c|c|c|c|c|c|}
\hline \multirow{2}{*}{$\begin{array}{l}\text { Credit (Max } \\
\text { Points) }\end{array}$} & \multicolumn{2}{|c|}{ Percent Averaged Score (PAS) } & \multirow{2}{*}{$p$-Value (Effect Size) } & \multirow{2}{*}{$\mathrm{SH}^{1}$} & \multirow{2}{*}{$\mathrm{SL}^{2}$} & \multirow{2}{*}{$\mathrm{DF}^{3}$} \\
\hline & Finland & Spain & & & & \\
\hline \multicolumn{7}{|c|}{ LEED Existing Buildings: Operations and Maintenance v3 (LEED-EB v3) } \\
\hline SSc1 (4) & 21 & 0 & $0.0448(2.31)^{a}$ & & & $\checkmark$ \\
\hline $\mathrm{SSc} 2(1)$ & 36 & 88 & $0.0046(-2.53)^{\mathrm{a}}$ & & & $\checkmark$ \\
\hline $\mathrm{SSc} 3(1)$ & 7 & 88 & $0.00001(-4.51)^{\mathrm{a}}$ & & & $\checkmark$ \\
\hline $\operatorname{SSc} 4(15)$ & 44 & 80 & $0.0001(-0.79)^{b}$ & & & $\checkmark$ \\
\hline $\operatorname{SSc} 5(1)$ & 43 & 69 & $0.2137(-1.08)^{\mathrm{a}}$ & $\checkmark$ & & \\
\hline SSc6 (1) & 7 & 13 & $0.7931(-0.62)^{\mathrm{a}}$ & & $\boldsymbol{V}$ & \\
\hline SSc7.1 (1) & 79 & 100 & $0.0448(-2.31)^{a}$ & & & $\checkmark$ \\
\hline SSc7.2 (1) & 0 & 13 & $0.347(-1.61)^{\mathrm{a}}$ & & $\checkmark$ & \\
\hline SSc8 (1) & 36 & 13 & $0.1450(1.36)^{a}$ & & $\checkmark$ & \\
\hline SS total (26) & 37 & 61 & $0.00002(-0.84)^{b}$ & & & $\checkmark$ \\
\hline \multicolumn{7}{|c|}{ LEED Existing Buildings: Operations and Maintenance v4 (LEED-EB v4) } \\
\hline LTc1 (15) & 72 & 89 & $0.0940(-0.35)^{b}$ & $\checkmark$ & & \\
\hline SSc1 (2) & 0 & 28 & $0.0005(-3.60)^{a}$ & & & $\checkmark$ \\
\hline SSc2 (3) & 0 & 0 & $0.5000(0.13)^{\mathrm{a}}$ & & $\checkmark$ & \\
\hline $\mathrm{SSc} 3(2)$ & 36 & 53 & $0.0183(-2.65)^{a}$ & & & $\checkmark$ \\
\hline $\mathrm{SSc} 4(1)$ & 79 & 75 & $0.8373(0.20)^{\mathrm{a}}$ & $\checkmark$ & & \\
\hline $\operatorname{SSc} 5(1)$ & 57 & 54 & $0.3830(0.54)^{\mathrm{a}}$ & $\checkmark$ & & \\
\hline SSc6 (1) & 0 & 38 & $0.0118(-2.89)^{\mathrm{a}}$ & & & $\checkmark$ \\
\hline SS total (10) & 21 & 32 & $0.0107(-0.52)^{b}$ & & & $\checkmark$ \\
\hline
\end{tabular}

For SSc7.1 (Heat Island Reduction-Non-roof in version 3) and SSc3 (Heat Island Reduction in version 4), Spain had a higher PAS than Finland, with 100 versus 79 in SSc7.1 (version 3) and 53 versus 36 in SSc3 (version 4). Spain also showed better results than Finland for the two additional credits of version 3 of the certification, namely SSc2 (Building Exterior and Hardscape Management Plan) and SSc3 (Integrated Pest Management, Erosion Control, and Landscape Management Plan (LMP)).

In terms of Light Pollution Reduction (SSc8 in version 3 and SSc4 in version 4), Finland's PASs increased from 36 to 79, and Spain's PASs increased from 13 to 75. However, for SSc6 (Stormwater Quantity Control in version 3), the PASs were 7 and 13 for Finland and Spain, respectively, while for SSc2 (Rainwater Management in version 4), the PASs were zero for both countries.

Thus, with regards to version 3 of the certification, Finland and Spain mostly had different achievements for credits, with only one similarly high credits achieved and three similarly low credits achieved (Table 3). However, considering version 4 of the certification, less than half of the credits were differently achieved, with increasing numbers of similarly high credits achieved and decreasing 
numbers of similarly low credits achieved. For almost all of these differently achieved credits (in both versions of the certification), Spain performed better than Finland. Thus, in the SS category (in versions 3 and 4), Spain presented better achievements than Finland.

\subsection{Water Efficiency (WE) Credits and Category}

Table 5 presents the results for the WE credits and categories. For the indoor-related credits, Finland improved its achievements, changing from PAS $=77$ (WEc2, Additional Indoor Plumbing Fixture and Fitting Efficiency, version 3) to PAS = 96 (WEc4, Indoor Water Use Reduction, version 4), becoming equal to Spain's PAS (95). For the outdoor water saving issue, Spain improved its achievement from PAS = 13 (WEc3, Water Efficient Landscaping, version 3) to PAS = 72 (WEc1, Outdoor Water Use Reduction, version 4), thereby approaching Finland's PAS (100).

Table 5. Statistical analysis of achieved points in the Water Efficiency (WE) credits for LEED-EB v3 and v4 large office-type projects in Finland and Spain.

\begin{tabular}{|c|c|c|c|c|c|c|}
\hline \multirow{2}{*}{$\begin{array}{l}\text { Credit (Max } \\
\text { Points) }\end{array}$} & \multicolumn{2}{|c|}{ Percent Averaged Score (PAS) } & \multirow{2}{*}{$p$-Value (Effect Size) } & \multirow{2}{*}{$\mathrm{SH}^{1}$} & \multirow{2}{*}{$\mathrm{SL}^{2}$} & \multirow{2}{*}{$\mathrm{DF}^{3}$} \\
\hline & Finland & Spain & & & & \\
\hline \multicolumn{7}{|c|}{ LEED Existing Buildings: Operations and Maintenance v3 (LEED-EB v3) } \\
\hline WEc1 (2) & 50 & 81 & $0.0133(-0.49)^{b}$ & \multirow{5}{*}{\multicolumn{2}{|c|}{$\boldsymbol{\nu}$}} & $\checkmark$ \\
\hline WEc2 (5) & 77 & 99 & $0.0081(-0.46)^{b}$ & & & $\checkmark$ \\
\hline WEc3 (5) & 86 & 13 & $0.00002(0.81)^{b}$ & & & $\checkmark$ \\
\hline WEc4 (2) & 0 & 13 & $0.1328(-0.25)^{a}$ & & & \\
\hline WE total (14) & 65 & 53 & $0.0180(0.50)^{b}$ & & & $\checkmark$ \\
\hline \multicolumn{7}{|c|}{ LEED Existing Buildings: Operations and Maintenance v4 (LEED-EB v4) } \\
\hline WEc1 (2) & 100 & 72 & $0.0613(0.31)^{b}$ & $\checkmark$ & \\
\hline WEc2 (3) & 29 & 19 & $0.5369(0.55)^{\mathrm{a}}$ & & \multicolumn{2}{|l|}{$\boldsymbol{v}$} \\
\hline WEc3 (2) & 11 & 25 & $0.0996(-1.30)^{\mathrm{a}}$ & & \multicolumn{2}{|l|}{$\checkmark$} \\
\hline WEc4 (5) & 96 & 95 & $1.0000(0.04)^{b}$ & \multicolumn{3}{|l|}{$\checkmark$} \\
\hline WE total (12) & 65 & 60 & $0.3943(0.18)^{b}$ & \multicolumn{3}{|l|}{$\checkmark$} \\
\hline
\end{tabular}

In contrast, for WEc1 (Water Performance Measurement in version 3) and WEc3 (Water Metering in version 4), the version-related improvement tendency was reversed. Namely, the PASs of Finland and Spain decreased between version 3 (50 and 81, respectively) to version 4 (11 and 25, respectively). For WEc4 (Cooling Tower Water Management in version 3) and WEc2 (Cooling Tower Water Use in version 4), Finland and Spain performed in a modest way. From version 3 to version 4 , there was in increase from PAS $=0$ to PAS $=29$ (Finland) and PAS $=13$ to PAS $=19$ (Spain).

Overall (see Table 4), three differently achieved credits and one similarly low credit were revealed for Finland's and Spain's LEED-EB v3 credits. As a result, in this version, Finland outperformed Spain in the WE category. However, in LEED-EB v4, WE credit achievement was improved, with two similarly high credit achievements in both Finland and Spain. Thus, in this version, both Finland and Spain had similarly high achievements in the WE category.

\subsection{Energy and Atmosphere (EA) Credits and Category}

Table 6 shows the results for the EA credits and categories. EAc4 (On-Site and Off-Site Renewable Energy in version 3) and EAc6 (Renewable Energy in version 4) were of low priority for both countries (PAS $=25-27$ for both countries), with the only exception being Spain's PAS for the LEED-EB v3 certification (48). In contrast, the issue of operational energy saving was of high priority for both 
countries. This was correct for both versions of the certification, showing PASs of 92 (Finland) and 87 (Spain) in version 3 (EAc1, Optimize Energy Efficiency Performance) and 91 (Finland) and 85 (Spain) in version 4 (EAc8, Optimize Energy Performance).

Table 6. Statistical analysis of achieved points in the Energy and Atmosphere (EA) credits for LEED-EB v3 and v4 large office-type projects in Finland and Spain.

\begin{tabular}{|c|c|c|c|c|c|c|}
\hline \multirow{2}{*}{$\begin{array}{l}\text { Credit (Max } \\
\text { Points) }\end{array}$} & \multicolumn{2}{|c|}{ Percent Averaged Score (PAS) } & \multirow{2}{*}{$p$-Value (Effect Size) } & \multirow{2}{*}{$\mathrm{SH}^{1}$} & \multirow{2}{*}{$\mathrm{SL}^{2}$} & \multirow{2}{*}{$\mathrm{DF}^{3}$} \\
\hline & Finland & Spain & & & & \\
\hline \multicolumn{7}{|c|}{ LEED Existing Buildings: Operations and Maintenance v3 (LEED-EB v3) } \\
\hline EAc1 (18) & 92 & 87 & $0.4976(0.14)^{b}$ & $\boldsymbol{V}$ & & \\
\hline EAc2.1 (2) & 100 & 81 & $0.1586(2.02)^{a}$ & $\boldsymbol{\sim}$ & & \\
\hline EAc2.2 (2) & 100 & 56 & $0.0045(3.13)^{a}$ & & & $\checkmark$ \\
\hline EAc2.3 (2) & 14 & 25 & $0.5172(-0.69)^{\mathrm{a}}$ & & $\boldsymbol{V}$ & \\
\hline EAc3.1 (1) & 100 & 19 & $0.00002(4.72)^{\mathrm{a}}$ & & & $\checkmark$ \\
\hline EAc3.2 (2) & 11 & 22 & $0.3016(-1.00)^{\mathrm{a}}$ & & $\boldsymbol{V}$ & \\
\hline EAc4 (6) & 27 & 48 & $0.3128(-0.21)^{b}$ & & $\checkmark$ & \\
\hline EAc5 (1) & 71 & 50 & $0.2094(0.92)^{\mathrm{a}}$ & $\boldsymbol{\sim}$ & & \\
\hline EAc6 (1) & 93 & 88 & $0.7931(0.62)^{a}$ & $\boldsymbol{V}$ & & \\
\hline EA total (35) & 72 & 68 & $0.1472(0.31)^{b}$ & $\boldsymbol{v}$ & & \\
\hline \multicolumn{7}{|c|}{ LEED Existing Buildings: Operations and Maintenance v4 (LEED-EB v4) } \\
\hline EAc1 (2) & 100 & 100 & $0.5000(-0.13)^{\mathrm{a}}$ & $\checkmark$ & & \\
\hline EAc2 (2) & 100 & 75 & $0.0697(2.35)^{\mathrm{a}}$ & $\checkmark$ & & \\
\hline EAc3 (3) & 50 & 0 & $0.0008(3.50)^{\mathrm{a}}$ & & & $\checkmark$ \\
\hline EAc4 (2) & 29 & 6 & $0.1009(1.79)^{\mathrm{a}}$ & & $\checkmark$ & \\
\hline EAc5 (3) & 0 & 2 & $0.7333(-1.03)^{\mathrm{a}}$ & & $\checkmark$ & \\
\hline EAc6 (5) & 26 & 25 & $0.8922(0.04)^{b}$ & & $\checkmark$ & \\
\hline EAc7 (1) & 64 & 50 & $0.1173(1.10)^{\mathrm{a}}$ & $\checkmark$ & & \\
\hline EAc8 (20) & 91 & 85 & $0.1129(0.33)^{b}$ & $\checkmark$ & & \\
\hline EA total (38) & 69 & 59 & $0.0013(0.66)^{b}$ & & & $\checkmark$ \\
\hline
\end{tabular}

The PASs of the building commissioning analysis issue were very high, with scores of 100 and 81 in Finland and Spain, respectively, for EAc2.1 (Existing Building Commissioning-Investigation and Analysis, version 3), and 100 in both Finland and Spain for EAc1 (Existing Building Commissioning-Analysis, version 4). For the building commissioning implementation issue, Finland $($ PAS $=100)$ outperformed Spain $($ PAS $=56)$ in version 3 (EAc2.2, Existing Building Commissioning-Implementation). However, in version 4 of the certification (EAc2, Existing Building Commissioning-Implementation), Spain improved its achievement (PAS $=75)$, approaching Finland's PAS (100). Only the ongoing commissioning issue (EAc2.3 in version 3 and EAc3 in version 4 of the certification) had mostly low PASs in both countries (0-25), with the exception of Finland's PAS in version 4 of the certification (50).

For EAc5 (Enhanced Refrigerant Management in version 3) and EAc7 (Enhanced Refrigerant Management version 4), the PASs were between 50 and 71. Lastly, the metering issue for EAc3.1 (Performance Measurement-Building Automation System) and EAc3.2 (Performance Measurement-System Level Metering) in version 3 and EAc4 (Advanced Energy Metering) and EAc5 (Demand Response) in version 4 of the certification performed poorly in both Finland and Spain (PASs = 0-29), with the exception of Finland's high PAS in version 3 with EAc3.1 (100).

Such EA credit achievements resulted in similar patterns for their distributions in three groups of achievements in versions 3 and 4 of the certifications (Table 5). In particular, most credits were 
achieved similarly in both Finland and Spain, with almost equal numbers of credits achieved similarly high as to those achieved similarly low.

\subsection{Materials and Resources (MR) Credits and Category}

Table 7 shows the results for the MR credits and categories. In both versions of the certification, only MRc4 (Sustainable Purchasing-Reduced Mercury in Lamps in version 3) and MRc3 (Purchasing-Lamps in version 4), as well as MRc6 (Solid Waste Management-Waste Stream Audit in version 3) and MRc1 (Solid Waste Management-Ongoing in version 4), had high PASs, 63-94, in Finland and Spain. For two additional credits, MRc7 (Solid Waste Management-Ongoing Consumables in version 3) and MRc2 (Solid Waste Management-Facility Maintenance in version 4), Finland achieved PASs of 93 and 36 for versions 3 and 4, respectively, while Spain achieved PASs of 25 and 6 for versions 3 and 4, respectively.

Table 7. Statistical analysis of achieved points in the Materials and Resources (MR) credits for LEED-EB v3 and v4 large office-type projects in Finland and Spain.

\begin{tabular}{|c|c|c|c|c|c|c|}
\hline \multirow{2}{*}{$\begin{array}{l}\text { Credit (Max } \\
\text { Points) }\end{array}$} & \multicolumn{2}{|c|}{ Percent Averaged Score (PAS) } & \multirow{2}{*}{$p$-Value (Effect Size) } & \multirow{2}{*}{$\mathrm{SH}^{1}$} & \multirow{2}{*}{$\mathrm{SL}^{2}$} & \multirow{2}{*}{$\mathrm{DF}^{3}$} \\
\hline & Finland & Spain & & & & \\
\hline \multicolumn{7}{|c|}{ LEED Existing Buildings: Operations and Maintenance v3 (LEED-EB v3) } \\
\hline MRc1 (1) & 0 & 0 & $0.5000(0.13)^{a}$ & & $\checkmark$ & \\
\hline MRc2.1 (1) & 21 & 19 & $0.8284(0.17)^{a}$ & & $\checkmark$ & \\
\hline MRc2.2 (1) & 7 & 0 & $0.2333(1.30)^{\mathrm{a}}$ & & $\checkmark$ & \\
\hline $\operatorname{MRc} 3(1)$ & 7 & 13 & $0.7931(-0.62)^{\mathrm{a}}$ & & $\checkmark$ & \\
\hline MRc4 (1) & 93 & 69 & $0.1240(1.78)^{\mathrm{a}}$ & $\checkmark$ & & \\
\hline MRc5 (1) & 0 & 0 & $0.5000(0.13)^{\mathrm{a}}$ & & $\checkmark$ & \\
\hline MRc6 (1) & 93 & 94 & $0.7425(-0.14)^{\mathrm{a}}$ & $\checkmark$ & & \\
\hline MRc7 (1) & 93 & 25 & $0.0001(3.66)^{\mathrm{a}}$ & & & $\boldsymbol{V}$ \\
\hline MRc8 (1) & 29 & 38 & $0.5756(-0.41)^{\mathrm{a}}$ & & $\checkmark$ & \\
\hline MRc9 (1) & 0 & 13 & $0.3471(-1.61)^{\mathrm{a}}$ & & $\checkmark$ & \\
\hline MR total (10) & 34 & 27 & $0.0819(0.36)^{b}$ & & $\checkmark$ & \\
\hline \multicolumn{7}{|c|}{ LEED Existing Buildings: Operations and Maintenance v4 (LEED-EB v4) } \\
\hline MRc1 (2) & 86 & 63 & $0.1632(1.28)^{\mathrm{a}}$ & $\checkmark$ & & \\
\hline MRc2 (2) & 36 & 6 & $0.0455(2.12)^{\mathrm{a}}$ & & & $\checkmark$ \\
\hline MRc3 (1) & 86 & 94 & $0.4069(-0.92)^{\mathrm{a}}$ & $\checkmark$ & & \\
\hline MRc4 (1) & 0 & 0 & $0.5000(0.13)^{a}$ & & $\nu$ & \\
\hline MRc5 (2) & 36 & 38 & $0.3512(-0.81)^{\mathrm{a}}$ & & $\checkmark$ & \\
\hline MR total (8) & 45 & 38 & $0.1853(0.28)^{b}$ & & $\nu$ & \\
\hline
\end{tabular}

All other sustainable purchasing credits, such as MRc1 (Sustainable Purchasing-Ongoing Consumables), MRc2.1 (Sustainable Purchasing_Electric-Powered Equipment), MRc2.2 (Sustainable Purchasing - Furniture), and MRc3 (Sustainable Purchasing-Facility Alterations and Additions) in version 3 and MRc4 (Purchasing-Ongoing) and MRc5 (Purchasing-Facility Maintenance) in version 4, had low PASs (0-38) in both Finland and Spain.

As a result, in both certification versions, Finland and Spain performed similarly low in the MR category, achieving similarly low PASs (for Finland, PASs of 34 and 45 in versions 3 and 4, respectively, and for Spain, PASs of 27 and 38 in versions 3 and 4, respectively). 


\subsection{Indoor Environmental Quality $(E Q)$ Credits and Category}

Table 8 shows the results for the EQ credits and categories. Only one credit, EQc2.4 (Daylight and Views), of the 15 total EQ credits was achieved to a similarly high degree (PAS $=64$ and PAS $=$ 63 in Finland and Spain, respectively) in version 3 of the certification. Interestingly, in version 3, for EQc2.1 (Occupant Comfort-Occupant Survey) and EQc3.2 (Green Cleaning-Custodial Effectiveness Assessment), Finland showed better results than Spain (PAS $=71$ and PAS $=79$ for Finland and PAS = 38 and PAS = 44 for Spain, respectively). However, in EQc1.1 (Indoor Air Quality Management Program) and EQc3.6 (Green Cleaning-Indoor Integrated Pest Management) in version 3, Spain showed better results than Finland (PAS $=69$ and PAS $=63$ for Spain and PAS $=21$ and PAS $=21$ for Finland, respectively).

Table 8. Statistical analysis of achieved points in the Indoor Environmental Quality (EQ) credits for LEED-EB v3 and v4 large office-type projects in Finland and Spain.

\begin{tabular}{|c|c|c|c|c|c|c|}
\hline \multirow{2}{*}{$\begin{array}{l}\text { Credit (Max } \\
\text { Points) }\end{array}$} & \multicolumn{2}{|c|}{ Percent Averaged Score (PAS) } & \multirow{2}{*}{$p$-Value (Effect Size) } & \multirow{2}{*}{$\mathrm{SH}^{1}$} & \multirow{2}{*}{$\mathrm{SL}^{2}$} & \multirow{2}{*}{$\mathrm{DF}^{3}$} \\
\hline & Finland & Spain & & & & \\
\hline \multicolumn{7}{|c|}{ LEED Existing Buildings: Operations and Maintenance v3 (LEED-EB v3) } \\
\hline EQc1.1 (1) & 21 & 69 & $0.0081(-2.09)^{\mathrm{a}}$ & & & $\checkmark$ \\
\hline EQc1.2 (1) & 0 & 0 & $0.5000(0.13)^{a}$ & & $\checkmark$ & \\
\hline EQc1.3 (1) & 93 & 56 & $0.0258(2.31)^{\mathrm{a}}$ & & & $\boldsymbol{\nu}$ \\
\hline EQc1.4 (1) & 100 & 50 & $0.0016(3.37)^{a}$ & & & $\boldsymbol{\nu}$ \\
\hline EQc1.5 (1) & 14 & 63 & $0.0064(-2.30)^{\mathrm{a}}$ & & & $\checkmark$ \\
\hline EQc2.1 (1) & 71 & 38 & $0.0537(1.43)^{a}$ & & & $\checkmark$ \\
\hline EQc2.2 (1) & 57 & 0 & $0.0003(3.76)^{\mathrm{a}}$ & & & $\checkmark$ \\
\hline EQc2.3 (1) & 7 & 0 & $0.2333(1.30)^{a}$ & & $\boldsymbol{v}$ & \\
\hline EQc2.4 (1) & 64 & 63 & $0.8533(0.08)^{\mathrm{a}}$ & $\checkmark$ & & \\
\hline EQc3.1 (1) & 43 & 38 & $0.8610(0.22)^{\mathrm{a}}$ & & $\checkmark$ & \\
\hline EQc3.2 (1) & 79 & 44 & $0.0476(1.55)^{\mathrm{a}}$ & & & $\checkmark$ \\
\hline EQc3.3 (1) & 71 & 13 & $0.0015(2.86)^{\mathrm{a}}$ & & & $\checkmark$ \\
\hline EQc3.4 (1) & 14 & 31 & $0.3016(-1.00)^{\mathrm{a}}$ & & & \\
\hline EQc3.5 (1) & 93 & 31 & $0.0004(3.35)^{a}$ & & & $\checkmark$ \\
\hline EQc3.6 (1) & 21 & 63 & $0.0208(-1.81)^{\mathrm{a}}$ & & & $\nu$ \\
\hline EQ total (15) & 50 & 37 & $0.0773(0.38)^{b}$ & & & $\checkmark$ \\
\hline \multicolumn{7}{|c|}{ LEED Existing Buildings: Operations and Maintenance v4 (LEED-EB v4) } \\
\hline EQc1 (2) & 100 & 94 & $0.7333(1.03)^{\mathrm{a}}$ & $\checkmark$ & & \\
\hline EQc2 (2) & 100 & 47 & $0.0697(2.35)^{\mathrm{a}}$ & & & $\checkmark$ \\
\hline EQc3 (1) & 0 & 6 & $0.7333(-1.03)^{\mathrm{a}}$ & & $\checkmark$ & \\
\hline EQc4 (2) & 0 & 3 & $0.7333(-1.03)^{\mathrm{a}}$ & & $\checkmark$ & \\
\hline EQc5 (4) & 4 & 47 & $0.0001(-3.66)^{\mathrm{a}}$ & & & $\checkmark$ \\
\hline EQc6 (1) & 93 & 63 & $0.0584(2.05)^{a}$ & $\checkmark$ & & \\
\hline EQc7 (1) & 71 & 52 & $0.2094(0.92)^{\mathrm{a}}$ & $\checkmark$ & & \\
\hline EQc8 (1) & 0 & 50 & $0.0016(-3.37)^{\mathrm{a}}$ & & & $\checkmark$ \\
\hline EQc9 (2) & 79 & 94 & $0.2092(-1.41)^{\mathrm{a}}$ & $\checkmark$ & & \\
\hline EQc10 (1) & 79 & 75 & $0.8373(0.20)^{a}$ & $\checkmark$ & & \\
\hline EQ total (17) & 48 & 49 & $0.3185(-0.21)^{b}$ & & $\checkmark$ & \\
\hline
\end{tabular}

In version 4, Spain increased their achievement in EQc10 (Occupant Comfort Survey) and EQc6 (Green Cleaning-Custodial Effectiveness Assessment), while Finland increased their achievement in EQc1 (Indoor Air Quality Management Program) and EQc9 (Integrated Pest Management). In this way, in version 4 , these four credits resulted in similarly high achievements for both Finland and Spain 
(PAS $=63-100)$. In addition, EQc7 (Green Cleaning_Products and Materials) also performed well in Finland and Spain, with high PASs of 71 and 52, respectively. Thus, in version 4, 5 of the 10 total credits had high PASs (52-100 for both countries).

EQc2.3 (Occupant Comfort-Thermal Comfort Monitoring in version 3) and EQc3 (Thermal Comfort in version 4) showed low PASs (0-7) in both countries. EQc1.2 (Indoor Air Quality Best Management Practices-Outdoor Air Delivery Monitoring) and EQc3.1 (Green CleaningHigh-Performance Cleaning Program) in version 3, as well as EQc4 (Interior Lighting) in version 4, also received low PASs (0-43) in both Finland and Spain.

Finally, in version 3 of the certification, Finland showed better results than Spain in 7 of the 10 total differently achieved credits. As a result, in the EQ category, Finland had a higher PAS than Spain (50 versus 37). However, in version 4 of the certification, Spain showed better results than Finland in two of the three differently achieved credits and, in seven credits, Finland and Spain had similar achievements. Therefore, both countries had very similar PASs of 48 and 49 in Finland and Spain, respectively (see Table 8).

\section{Discussion}

\subsection{Sustainable Sites and Location and Transportation Credits}

Public transportation is an issue that can be easily solved in highly urbanized countries through highly developed public transportation infrastructure [4]. Most of the analyzed projects were located in high-density cites such as Helsinki (Finland) or Madrid and Barcelona (Spain), as shown in Table 2. Thus, the high performance of both Finland and Spain, in terms of public transportation issues (SSc4 in version 3 and LTc1in version 4, Table 3), was expected.

The urban heat island (UHI) reduction issue (SSc7.1 in version 3 and SSc3 in version 3 and version 4, Table 3) was more popular in Spain than in Finland. This is because this issue has higher relevance in Spain's climate, which includes five weather groups: oceanic, continental, Mediterranean, semi-arid, and sub-tropical [43]. Such climates mainly have hot summers with high solar radiation. In particular, most projects considered in this study were located in Madrid (sub-tropical climate) and Barcelona (Mediterranean climate). In such climates, the UHI effect can reach $4.3^{\circ} \mathrm{C}$ at street level, increasing cooling demands and thus building energy consumption [44].

For the next two issues-light pollution reduction and rainwater management-similar approaches were found for Finland and Spain. The light pollution reduction issue (SSc8 in version 3 and SSc4 in version 4, Table 3) was improved in both Finland and Spain when changing from version 3 to version 4 .

However, the rainwater management issue (SSc6 in version 3 and SSc2 in version 4, Table 3) was found to be unattractive in both countries. Among the five aforementioned weather groups of Spain, only oceanic climates are characterized by high rainfall, whereas the other four climate groups (continental, Mediterranean, semi-arid, and sub-tropical) are characterized by limited or scarce rainfall [43]. Thus, the rainwater management issue is not exactly relevant to Spain. In contrast, temperatures and rainfall differ significantly in southern and northern Finland [45]. However, most of the projects considered in this study were located in Helsinki (humid continental climate) and Espoo (cold and temperate climate), both of which feature significant rainfall throughout the year. Thus, the rainwater management issue seems to be more relevant to Finland. However, as was noticed, this credit was given little to no attention in this country.

\subsection{Water Efficiency Credits}

Compared to version 3, in version 4 of the certification, the indoor (WEc4 in version 4) and outdoor (WEc1 in version 4) water saving issues were improved in both Finland and Spain (Table 4). This was despite the differences in the country-specific currents and potential water resources. Finland (northern Europe) has adequate sources for water supplies [46], whereas Spain (southern Europe) experiences high water stress [47]. It is expected that, in future decades, in northern Europe, precipitation patterns 
may change due to climate changes (e.g., more precipitation in winter and less precipitation in summer) [48], whereas in southern Europe, cumulative annual precipitation may decrease, especially in Mediterranean areas [47]. However, in terms of the water metering issue (WEc1 in version 3 and WEc3 in version 4), performance decreased from version 3 to version 4 (Table 4).

Finally, the cooling tower water saving issue (WEc4 in version 3 and WEc2 in version 4) performed poorly in both Finland and Spain (Table 4). Cooling tower water-related credits require the reduction of potable water for cooling towers and evaporative condensers [23,24]. Such heating, ventilation, and air conditioning (HVAC) systems are usually installed in large buildings [12] (p. 17). Thus, the low popularity of this issue in the large offices of both Finland and Spain seems surprising.

\subsection{Energy and Atmosphere Credits}

The renewable energy issue (EAc4 in version 3 and EAc6 in version 4) had low performance in both Finland and Spain (Table 5). This was surprising, as the share of renewable energy constitutes approximately one third of all produced energy in both countries. In Finland, renewable energy is produced by hydropower (28\%) and wind, solar, and biofuel (3\%) processes [49], whereas in Spain, it is produced by wind (19\%), solar (5\%), and biomass (11\%) processes [50].

In contrast, the issue of operational energy saving (EAc1 in version 3 and EAc8 in version 4) was of high priority in both countries (Table 5). Thus, large offices in Finland and Spain emphasized the operational energy saving issue. The operational energy saving issue depends on the decisions of building design teams to improve the building envelope (e.g., wall and roof-related insulation and thermal mass and window-related solar and thermal insulation) and HVAC efficiency [23,24]. In European countries, decreasing the operational energy of buildings has been strongly motivated by European Union-related development strategies for moving toward a low-carbon economy by 2050 [51]. In this respect, Finland has targeted the reduction of district heating fossil fuel-based emissions by $80 \%$ by 2050 [52]. In Spain, the basic energy efficiency document (energy conservation in the Spanish building code) restricts thermal conductivity (the U-value), among other parameters, to reduce operational energy in the building sector [53]. Therefore, it was not surprising that the issue of operational energy saving was of high priority in both countries for both versions of the certifications.

\subsection{Materials and Resources Credits}

In both versions of the certification, only the sustainable purchasing of lamps with reduced mercury (MRc4 and MR3 in versions 3 and 4, respectively) and solid waste management (MRc6 and MRc1 in versions 3 and 4, respectively) were popular issues in both Finland and Spain (Table 6). All other sustainable purchasing, with regards to ongoing consumables, electric-powered equipment, furniture, facility alterations, and ongoing and facility maintenance (MRc1, MRc2.1, MRc2.2, and MRc3 in version 3 and MRc4 and MRc5 in version 4) were considered to be unattractive issues by both Finland and Spain (Table 6). These sustainable purchasing criteria require purchasing goods that contain certain percentages of post-consumer, post-industrial, rapidly renewable, and salvageable components from on-site materials [23]. The replacement of virgin materials after manufacturing has always been an unattractive and difficult issue [54].

The MR category has also shown low achievement in other LEED systems and countries: LEED-NC v3 projects certified around the world; LEED-CI v3 and LEED-C and S projects certified in Turkey, Spain, and Italy; LEED-NC v3 projects certified in the U.S., China, Turkey, and Brazil; and LEED-CI v4 projects certified in the U.S. [2,5,14,55]. In all these LEED systems, MR credits have different formulations, depending on the type of certified building, such as newly constructed buildings, existing buildings, commercial interiors, or core and shell. Regardless, this category is generally not considered popular among building practitioners. This issue should attract the attention of LEED experts, in terms of encouraging the attractiveness of these credits in building communities. 


\subsection{Indoor Environmental Quality Credits}

Thermal comfort issues (EQc2.3 in version 3 and EQc3 in version 4) were considered unpopular in both Finland and Spain (Table 7). This credit requires installing systems for tracking thermal comfort by measuring air temperature and humidity in occupied spaces $[23,24]$. It seems that this credit should be important in large offices, due to the high density of occupied spaces. Thus, it was unexpected to see low interest in this issue in both countries.

The three additional issues of outdoor air delivery monitoring (EQc1.2 in version 3), a highperformance cleaning program (EQc3.1 in version 3), and interior lighting (EQc4 in version 4) were also unpopular in both Finland and Spain (Table 7). The outdoor air delivery monitoring issue involves the installation of monitoring systems to measure $\mathrm{CO}_{2}$ concentrations in occupied spaces. The high-performance cleaning program issue requires using sustainable cleaning materials with decreased contents of hazardous chemicals. Lastly, the interior lighting issue encourages the provision of high-quality lighting by installing individual lighting controls for building occupants [23,24]. These credits also seem to be important for densely occupied offices. Thus, it is interesting that both Finland and Spain paid little attention to these credits.

\section{Summary of Findings}

Table 9 shows the high-frequency credits that were very helpful for achieving LEED-EB v4 Gold certification in office-type buildings located in Finland and Spain.

Table 9. High-frequency credits in Finland and Spain for LEED-EB v4 Gold certification of large office-type projects.

\begin{tabular}{|c|c|c|}
\hline Category & Finland & Spain \\
\hline LT and SS & $\begin{array}{l}\text { LTc1 Alternative Transportation, SSc4 } \\
\text { Light Pollution Reduction, SSc5 Site } \\
\text { Management }\end{array}$ & $\begin{array}{l}\text { LTc1 Alternative Transportation, SSc3 } \\
\text { Heat Island Reduction, SSc4 Light } \\
\text { Pollution Reduction, SSc5 Site } \\
\text { Management }\end{array}$ \\
\hline WE & $\begin{array}{l}\text { WEc1 Outdoor Water Use Reduction, } \\
\text { WEc4 Indoor Water Use Reduction }\end{array}$ & $\begin{array}{l}\text { WEc1 Outdoor Water Use Reduction, } \\
\text { WEc4 Indoor Water Use Reduction }\end{array}$ \\
\hline EA & $\begin{array}{l}\text { EAc1 and EAc2 Existing Building } \\
\text { Commissioning-Analysis and } \\
\text { Implementation, EAc7 Enhanced } \\
\text { Refrigerant Management, EAc8 Optimize } \\
\text { Energy Performance }\end{array}$ & $\begin{array}{l}\text { EAc1 and EAc2 Existing Building } \\
\text { Commissioning-Analysis and } \\
\text { Implementation, EAc8 Optimize Energy } \\
\text { Performance }\end{array}$ \\
\hline MR & $\begin{array}{l}\text { MRc1 Solid Waste } \\
\text { Management-Ongoing, MRc3 } \\
\text { Purchasing-Lamps }\end{array}$ & $\begin{array}{l}\text { MRc1 Solid Waste } \\
\text { Management-Ongoing, MRc3 } \\
\text { Purchasing-Lamps }\end{array}$ \\
\hline EQ & $\begin{array}{l}\text { EQc1 Indoor Air Quality Management } \\
\text { Program, EQc2 Enhanced Indoor Air } \\
\text { Quality Strategies, EQc6 and EQc7 Green } \\
\text { Cleaning-Custodial Effectiveness } \\
\text { Assessment and Products and Materials, } \\
\text { EQc9 Integrated Pest Management, EQc10 } \\
\text { Occupant Comfort Survey }\end{array}$ & $\begin{array}{l}\text { EQc1 Indoor Air Quality Management } \\
\text { Program, EQc6 and EQc7 Green } \\
\text { Cleaning-Custodial Effectiveness } \\
\text { Assessment and Products and Materials, } \\
\text { EQc9 Integrated Pest Management, EQc10 } \\
\text { Occupant Comfort Survey }\end{array}$ \\
\hline
\end{tabular}

\section{Conclusions}

The transition from LEED version 3 to version 4 has provided more flexible rating systems, such that each country can use its own certification strategy at a local level. However, these strategies may not be unique to all building types. In this study, LEED-EB v3 and v4 Gold large office-type $\left(>6435 \mathrm{~m}^{2}\right)$ projects in Finland and Spain — countries with similar values in the European community, but different resources and climates-were analyzed.

The following trends were noticed in the shift from version 3 to version 4 LEED-EB Gold projects. In the LT, SS, WE, and EQ categories, the number of differently achieved credits between Finland 
and Spain decreased, whereas the number of similarly high credits of achievement in these countries increased. However, in the EA and MR categories, the number of similarly high and similarly low credits achieved between Finland and Spain remained almost the same. As a result, in version 4, the high-frequency credits in Finland and Spain for LEED-EB v4 Gold certification were almost the same (Table 9).

This suggests that the certification strategies for large offices in Finland and Spain were more similar than different. These results may be useful for LEED practitioners in Finland and Spain in order to facilitate the selection of appropriate strategies for LEED-EB v4 large offices.

In addition, it can be concluded that, when improving from LEED-EB version 3 to version 4, each of these countries had possibilities to improve their performance. Finland showed improvement in the transportation issue (LT), and Spain showed improvement in outdoor water reduction (WE) and indoor air quality management issues (EQ). This can be seen as empirical evidence that LEED-EB has become more flexible in the transition from version 3 to version 4 .

This study fills the gap associated with a lack of empirical research in LEED certification design, with a specific data collection framework aimed at reducing the impact of uncontrollable factors, such as different certification levels, different building types, and different building sizes. Thus, the certification strategies disclosed here can be considered as more robust than those recommended by previous studies, which did not account for such uncontrollable factors.

A limitation of this study is the current small number of LEED-EB v4 projects in Europe. In order to better develop certification strategies, more research is required, ideally taking into account the future accumulation of LEED projects in foreign countries.

Funding: This research received no external funding.

Conflicts of Interest: The author declares that there is no conflict of interest.

\section{Appendix A}

Table A1. Full LT and SS credit titles.

\begin{tabular}{c} 
Credit or Category \\
\hline LEED Existing Buildings: Operations and Maintenance v3 (LEED-EB v3) \\
SSc1 LEED Certified Design and Construction \\
SSc2 Building Exterior and Hardscape Management Plan \\
SSc3 Integrated Pest Management and Erosion Control and Landscape Management Plan \\
SSc4 Alternative Commuting Transportation \\
SSc5 Site Development-Protect or Restore Open Habitat \\
SSc6 Stormwater Quantity Control \\
SSc7.1 Heat Island Reduction-Nonroof \\
SSc7.2 Heat Island Reduction-Roof \\
SSc8 Light Pollution Reduction \\
SS Total \\
LEED Existing Buildings: Operations and Maintenance v4 (LEED-EB v4) \\
LTc1 Alternative Transportation \\
SSc1 Site Development-Protect or Restore Habitat \\
SSc2 Rainwater Management \\
SSc3 Heat Island Reduction \\
SSc4 Light Pollution Reduction \\
SSc5 Site Management \\
SSc6 Site Improvement Plan \\
SS Total \\
\hline
\end{tabular}


Table A2. Full WE credit titles.

\section{Credit or Category}

LEED Existing Buildings: Operations and Maintenance v3 (LEED-EB v3)

WEc1 Water Performance Measurement

WEc2 Additional Indoor Plumbing Fixture and Fitting Efficiency

WEc3 Water Efficient Landscaping

WEc4 Cooling Tower Water Management

WE Total

LEED Existing Buildings: Operations and Maintenance v4 (LEED-EB v4)

WEc1 Outdoor Water Use Reduction

WEc2 Cooling Tower Water Use

WEc3 Water Metering

WEc4 Indoor Water Use Reduction

WE Total

Table A3. Full EA credit titles.

\begin{tabular}{c} 
Credit or Category \\
\hline LEED Existing Buildings: Operations and Maintenance v3 (LEED-EB v3) \\
EAc1 Optimize Energy Efficiency Performance \\
EAc2.1 Existing Building Commissioning-Investigation and Analysis \\
EAc2.2 Existing Building Commissioning-Implementation \\
EAc2.3 Existing Building Commissioning-Ongoing Commissioning \\
EAc3.1 Performance Measurement-Building Automation System \\
EAc3.2 Performance Measurement-System Level Metering \\
EAc4 On-site and Off-site Renewable Energy \\
EAc5 Enhanced Refrigerant Management \\
EAc6 Emissions Reduction Reporting \\
EA Total \\
EAED Existing Buildings: Operations and Maintenance v4 (LEED-EB v4) \\
EAc1 Existing Building Commissioning-Analysis \\
EAc2 Existing Building Commissioning-Implementation \\
EAc3 Ongoing Commissioning \\
EAc4 Advanced Energy Metering \\
EAc5 Demand Response \\
EAc6 Renewable Energy \\
EAc7 Enhanced Refrigerant Management \\
EAc8 Optimize Energy Performance \\
EA Total \\
\hline
\end{tabular}


Table A4. Full MR credit titles.

\section{Credit or Category}

\begin{tabular}{c} 
Credit or Category \\
\hline LEED Existing Buildings: Operations and Maintenance v3 (LEED-EB v3) \\
MRc1 Sustainable Purchasing-Ongoing Consumables \\
MRc2.1 Sustainable Purchasing-Electric-Powered Equipment \\
MRc2.2 Sustainable Purchasing-Furniture \\
MRc3 Sustainable Purchasing-Facility Alterations and Additions \\
MRc4 Sustainable Purchasing-Reduced Mercury in Lamps \\
MRc5 Sustainable Purchasing-Food \\
MRc6 Solid Waste Management-Waste Stream Audit \\
MRc7 Solid Waste Management-Ongoing Consumables \\
MRc8 Solid Waste Management-Durable Goods \\
MRc9 Solid Waste Management-Facility Alterations and Additions \\
MR Total \\
LEED Existing Buildings: Operations and Maintenance v4 (LEED-EB v4) \\
MRc1 Solid Waste Management-Ongoing \\
MRc2 Solid Waste Management-Facility Maintenance \\
MRc3 Purchasing-Lamps \\
MRc4 Purchasing-Ongoing \\
MRc5 Purchasing-Facility Maintenance \\
MR Total
\end{tabular}

Table A5. Full EQ credit titles.

\begin{tabular}{|c|}
\hline Credit or Category \\
\hline LEED Existing Buildings: Operations and Maintenance v3 (LEED-EB v3) \\
\hline $\begin{array}{l}\text { EQc1.1 Indoor Air Quality Best Management Practices-IAQ Management Program } \\
\text { EQc1.2 Indoor Air Quality Best Management Practices-Outdoor Air Delivery Monitoring } \\
\text { EQc1.3 Indoor Air Quality Best Management Practices-Increased Ventilation } \\
\text { EQc1.4 Indoor Air Quality Best Management Practices-Reduce Particulates in Air Distribution } \\
\text { EQc1.5 Indoor Air Quality Best Management Practices-IAQ }{ }^{4} \text { Management for Facility Alterations and } \\
\text { Additions } \\
\text { EQc2.1 Occupant Comfort-Occupant Survey } \\
\text { EQc2.2 Controllability of Systems-Lighting } \\
\text { EQc2.3 Occupant Comfort-Thermal Comfort Monitoring } \\
\text { EQc2.4 Daylight and Views } \\
\text { EQc3.1 Green Cleaning-High Performance Cleaning Program } \\
\text { EQc3.2 Green Cleaning-Custodial Effectiveness Assessment } \\
\text { EQc3.4 Green Cleaning-Sustainable Cleaning Equipment } \\
\text { EQc3.3 Green Cleaning-Purchase of Sustainable Cleaning Products and Materials } \\
\text { EQc3.6 Green Cleaning-Indoor Integrated Pest Management } \\
\text { EQ Total }\end{array}$ \\
\hline LEED Existing Buildings: Operations and Maintenance v4 (LEED-EB v4) \\
\hline $\begin{array}{c}\text { EQc1 Indoor Air Quality Management Program } \\
\text { EQc2 Enhanced Indoor Air Quality Strategies } \\
\text { EQc3 Thermal Comfort } \\
\text { EQc4 Interior Lighting } \\
\text { EQc5 Daylight and Quality Views } \\
\text { EQc6 Green Cleaning-Custodial Effectiveness Assessment } \\
\text { EQc7 Green Cleaning-Products and Materials } \\
\text { EQc8 Green Cleaning-Equipment } \\
\text { EQc9 Integrated Pest Management } \\
\text { EQc10 Occupant Comfort Survey } \\
\text { EQ Total }\end{array}$ \\
\hline
\end{tabular}




\section{References}

1. Ade, R.; Rehm, M. The unwritten history of green building rating tools: A personal view from some of the 'founding fathers'. Build. Res. Inform. 2020, 48, 1-17. [CrossRef]

2. Wu, P.; Song, Y.Z.; Shou, W.C.; Chi, H.L.; Chong, H.Y.; Sutrisna, M. A comprehensive analysis of the credits obtained by LEED 2009 certified green buildings. Renew. Sustain. Energy Rev. 2017, 68 Pt 1, 370-379. [CrossRef]

3. Głuszak, M. Internationalization, Competiveness and Green Building Certification in Europe (Chapter 9). In Europeanization Processes from the Mesoeconomic Perspective: Industries and Policies, 2nd ed.; Stanek, P., Wach, K., Eds.; Cracow University of Economics: Kraków, Poland, 2015; pp. 173-191.

4. Suzer, O. A comparative review of environmental concern prioritization: LEED vs other major certification systems. J. Environ. Manag. 2015, 154, 266-283. [CrossRef] [PubMed]

5. Wu, P.; Song, Y.; Wang, J.; Wang, X.; Zhao, X.; He, Q. Regional Variations of Credits Obtained by LEED 2009 Certified Green Buildings-A Country Level Analysis. Sustainability 2018, 10, 20. [CrossRef]

6. Cheng, J.C.P.; Ma, L.J. A study of the relationship between credits in the LEEDEB\&OM green building rating system. IACSIT Int. J. Eng. Technol. 2013, 5, 438-442. [CrossRef]

7. Cheng, J.C.P.; Ma, L.J. A non-linear case-based reasoning approach for retrieval of similar cases and selection of target credits in LEED projects. Build. Environ. 2015, 93 Pt 2, 349-361. [CrossRef]

8. Pushkar, S. Sacrificial Pseudoreplication in LEED Cross-Certification Strategy Assessment: Sampling Structures. Sustainability 2018, 10, 1353. [CrossRef]

9. Gurgun, A.P.; Arditi, D. Assessment of energy credits in LEED-Certified Buildings based on certification levels and project ownership. Buildings 2018, 8, 29. [CrossRef]

10. Toğan, V.; Thomollari, X. Credit Success Rates of Certified Green Buildings in Turkey. Tek. Dergi 2020, 31, 10063-10084. [CrossRef]

11. Pushkar, S. A comparative analysis of Gold Leadership in Energy and Environmental Design for New Construction 2009 Certified projects in Finland, Sweden, Turkey, and Spain. Appl. Sci. 2018, 8, 1496. [CrossRef]

12. Yan, L. Energy Consumption Analyzes of Frequently-Used HVAC System Types in High Performance Office Buildings. Master's Thesis, The Graduate School College of Engineering, The Pennsylvania State University, State College, PA, USA, 2014; p. 86. Available online: File://C:/Users/user/Documents/Journal\%20Papers/ LEEDEBv4/After\%20review\%2010\%2006\%202020/9398.pdf (accessed on 21 October 2020).

13. Pham, D.H.; Kim, B.; Lee, J.; Ahn, A.C.; Ahn, Y. A comprehensive analysis: Sustainable trends and awarded LEED 2009 credits in Vietnam. Sustainability 2020, 12, 852. [CrossRef]

14. Pushkar, S. The effect of regional priority points on the performance of LEED 2009 certified buildings in Turkey, Spain, and Italy. Sustainability 2018, 10, 3364. [CrossRef]

15. Intelligent Energy Europe: Report on Specific Features of Public and Social Acceptance and Perception of Nearly Zero-Energy Buildings and Renewable Heating and Cooling in Europe with A Specific Focus on the Target Countries. Available online: http://www.entranze.eu/files/downloads/ENTRANZE_D2_6_Final_ version.pdf (accessed on 12 August 2020).

16. Evonne, M.; Laurie, B. Retrofitting commercial office buildings for sustainability: Tenants' perspectives. J. Prop. Invest. Financ. 2008, 26, 552-561. Available online: https://eprints.qut.edu.au/15428/1/15428.pdf (accessed on 21 October 2020).

17. Asadi, E.; da Silva, M.G.; Antunes, C.H.; Dias, L. Multi-objective optimization for building retrofit strategies: A model and an application. Energy Build. 2012, 44, 81-87. [CrossRef]

18. McArthur, J.J.; Jofeh, C.G.H. Portfolio retrofit evaluation: A methodology for optimizing a large number of building retrofits to achieve triple-bottom-line objectives. Sustain. Cities Soc. 2016, 27, 263-274. [CrossRef]

19. Dall'O, G.; Bruni, E.; Panza, A. Improvement of the Sustainability of Existing School Buildings According to the Leadership in Energy and Environmental Design (LEED) ${ }^{\circledR}$ Protocol: A Case Study in Italy. Energies 2013, 6, 6487-6507. [CrossRef]

20. Sun, X.; Gou, Z.; Lu, Y.; Tao, Y. Strengths and Weaknesses of Existing Building Green Retrofits: Case Study of a LEED EBOM Gold Project. Energies 2018, 11, 1936. [CrossRef]

21. Mazzola, E.; Mora, T.D.; Peron, F.; Romagnoni, P. An Integrated Energy and Environmental Audit Process for Historic Buildings. Energies 2019, 12, 3940. [CrossRef] 
22. Jin, X.; Meng, C.; Wang, Q.; Wei, J.S.; Zhang, L. A study of the green retrofit industry chain. Sustain. Cities Soc. 2014, 13, 143-147. [CrossRef]

23. LEED-EBv4 2019. Available online: http://greenguard.org/uploads/images/ LEEDv4forBuildingOperationsandMaintenanceBallotVersion.pdf (accessed on 10 September 2020).

24. LEED-EBv3 2014. Available online: https://energy.nv.gov/uploadedFiles/energynvgov/content/Programs/ 2009_EBOM.pdf (accessed on 12 September 2020).

25. USGBC Projects Site. Available online: https://www.usgbc.org/projects (accessed on 21 October 2020).

26. GBIG Green Building Data. Available online: http://www.gbig.org (accessed on 21 October 2020).

27. Pushkar, S. LEED-CI V3 and V4 Silver and Gold Projects in China and the U.S. Appl. Sci. 2020, 10, 4524. [CrossRef]

28. Pushkar, S. Evaluating LEED commercial interior (LEED-CI) projects under the LEED transition from v3 to v4: The differences between China and the US. Heliyon 2020, 6, e04701. [CrossRef] [PubMed]

29. Pham, D.H.; Kim, B.; Lee, J.; Ahn, Y. An Investigation of the Selection of LEED Version 4 Credits for Sustainable Building Projects. Appl. Sci. 2020, 10, 7081. [CrossRef]

30. Bland, J.M.; Altman, D.G. The odds ratio. BMJ 2000, 320, 1468. [CrossRef]

31. Routledge, R.D. Resolving the conflict over Fisher's exact test. Can. J. Statist. 1992, 20, 201-209. Available online: https://www.jstor.org/stable/3315468 (accessed on 14 September 2020). [CrossRef]

32. Cliff, N. Dominance statistics: Ordinal analyses to answer ordinal questions. Psychol. Bull. 1993, 114, 494-509. [CrossRef]

33. Bergmann, R.; Ludbrook, J.; Spooren, W.P.J.M. Different outcomes of the Wilcoxon-Mann-Whitney test from different statistics packages. Am. Stat. 2000, 54, 72-77. Available online: http://www.jstor.org/stable/2685616 (accessed on 5 September 2020).

34. Chi, B.; Lu, W.; Ye, M.; Bao, Z.; Zhang, X. Construction waste minimization in green building: A comparative analysis of LEED-NC 2009 certified projects in the US and China. J. Clean. Prod. 2020, 256, 120749. [CrossRef]

35. Haddock, C.K.; Rindskopf, W.R.; Shadish, W.R. Using odds ratios as effect sizes for meta-analysis of dichotomous data: A primer on methods and issues. Psychol. Methods 1998, 3, 339-353. [CrossRef]

36. Chen, H.; Cohen, P.; Chen, S. How Big is a Big Odds Ratio? How big is a big odds ratio? Interpreting the magnitudes of odds ratios in epidemiological studies. Commun. Stat. Simul. Comput. 2010, 39, 860-864. [CrossRef]

37. Romano, J.; Corragio, J.; Skowronek, J. Appropriate statistics for ordinal level data: Should we really be using t-test and Cohen's d for evaluating group differences on the NSSE and other surveys? In Proceedings of the Annual Meeting of the Florida Association of Institutional Research, Cocoa Beach, FL, USA, 1-3 February 2006; Florida Association for Institutional Research: Cocoa Beach, FL, USA, 2006; pp. 1-33.

38. Cohen, J. A power primer. Psychol. Bull. 1992, 112, 155-159. [CrossRef]

39. Durlak, J.A. How to select, calculate, and interpret effect sizes. J. Pediatr. Psychol. 2009, 34, 917-928. [CrossRef] [PubMed]

40. Volker, M.A. Reporting effect size estimates in school psychology research. Psychol. Sch. 2006, 43, 653-672. [CrossRef]

41. Hurlbert, S.H.; Lombardi, C.M. Final collapse of the Neyman-Pearson decision theoretic framework and rise of the neoFisherian. Ann. Zool. Fenn. 2009, 46, 311-349. Available online: https://www.jstor.org/stable/23736900 (accessed on 2 September 2020). [CrossRef]

42. Beninger, P.G.; Boldina, I.; Katsanevakis, S. Strengthening statistical usage in marine ecology. J. Exp. Mar. Biol. Ecol. 2012, 426-427, 97-108. [CrossRef]

43. Zarco-Soto, I.M.; Zarco-Perinan, P.J.; Sanchez-Duran, R. Influence of climate on energy consumption and $\mathrm{CO}_{2}$ emissions: The case of Spain. Environ. Sci. Pollut. Res. 2020, 27, 15645-15662. [CrossRef]

44. Salvati, A.; Roura, H.C.; Cecere, C. Assessing the urban heat island and its energy impact on residential buildings in Mediterranean climate: Barcelona case study. Energy Build. 2017, 146, 38-54. [CrossRef]

45. Pedretti, D.; Irannezhad, M. Non-stationary peaks-over-threshold analysis of extreme precipitation events in Finland, 1961-2016. Int. J. Climatol. 2019, 39, 1128-1143. [CrossRef]

46. Laitinen, J.; Antikainen, R.; Hukka, J.J.; Katko, T.S. Water Supply and Sanitation in a Green Economy Society: The Case of Finland. Publ. Work. Manag. Policy 2020, 25, 33-50. [CrossRef]

47. Estrela, T.; Pérez-Martin, M.A.; Vargas, E. Impacts of climate change on water resources in Spain. Hydrol. Sci. J. 2012, 57, 1154-1167. [CrossRef] 
48. Nykvist, B.; Borgström, S.; Boyd, E. Assessing the adaptive capacity of multi-level water governance: Ecosystem services under climate change in Mälardalen region, Sweden. Reg. Environ. Chang. 2017, 8, 2359-2371. [CrossRef]

49. Heiskanen, E.; Jalas, M.; Juntune, J.K.; Nissila, H. Small streams, diverse sources: Who invests in renewable energy in Finland during the financial downturn? Energy Policy 2017, 106, 191-200. [CrossRef]

50. Gómez-Calvet, R.; MartÍnez-Duart, J.M.; Calle, S.S. Present state and perspectives of variable renewable energies in Spain. Eur. Phys. J. Plus 2018, 133, 126. [CrossRef]

51. European Commission. A Roadmap for Moving to a Competitive Low Carbon Economy in 2050. Brussels, Belgium. 2011. Available online: http://ec.europa.eu/clima/policies/roadmap/index_en.htm (accessed on 21 October 2020).

52. Rehman, H.U.; Hirvonen, J.; Jokisalo, J.; Kosonen, R.; Sirén, K. EU Emission Targets of 2050: Costs and CO Emissions Comparison of Three Different Solar and Heat Pump-Based Community-Level District Heating Systems in Nordic Conditions. Energies 2020, 13, 4167. [CrossRef]

53. Bienvenido-Huertas, D.; Rubio-Bellido, C.; Pulido-Arcas, J.A.; Pérez-Fargallo, A. Towards the implementation of periodic thermal transmittance in Spanish building energy regulation. J. Build. Eng. 2020, 31, 101402. [CrossRef]

54. Pushkar, S.; Verbitsky, O. LEED-NCv3 Silver and Gold certified projects in the US: An observational study. J. Green Build. 2018, 13, 67-83. [CrossRef]

55. Pushkar, S. LEED-CIv4 Commercial Interiors: United States (2014-2019). Sustainability 2020, 12, 69. [CrossRef]

Publisher's Note: MDPI stays neutral with regard to jurisdictional claims in published maps and institutional affiliations.

(C) 2020 by the author. Licensee MDPI, Basel, Switzerland. This article is an open access article distributed under the terms and conditions of the Creative Commons Attribution (CC BY) license (http://creativecommons.org/licenses/by/4.0/). 